\title{
PREVENÇÃO DE LESÕES POR PRESSÃO NA PRONAÇÃO DE PACIENTES COVID-19: CONSTRUÇÃO DE UMA NARRATIVA GRÁFICA
}

\author{
Daniela Soldera ${ }^{1, *}$ (D), Cilene Fernandes Soares² (D), Juliana Balbinot Reis Girondi ${ }^{3}$ (D),

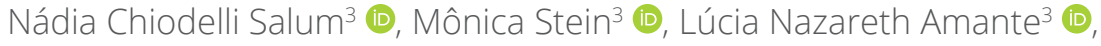 \\ Luciara Fabiane Sebold ${ }^{3}$ (D), Gisele Martins Miranda ${ }^{4}$ (1)
}

\begin{abstract}
RESUMO
Objetivo: construir e validar o conteúdo de uma narrativa gráfica sobre a prevenção de Lesão por Pressão na posição prona em pacientes acometidos pelo Coronavírus. Método: estudo metodológico descritivo. Para a construção da narrativa gráfica, foi realizada a revisão de escopo. A validação de conteúdo da narrativa foi realizada por enfermeiros atuantes em unidades de terapia intensiva respiratória estomaterapeutas de Santa Catarina, os quais responderam a um formulário no Googleforms $®$ com opções de resposta de discordo a concordo. A análise de dados foi realizada por meio do índice de Validade de Conteúdo, considerando adequado $>0,80 \%$. Resultados: os cuidados elencados para compor a narrativa foram: avaliação da pele; manutenção da pele limpa e hidratada; utilização de dispositivos de redistribuição de pressão; utilização de espumas de silicone, filmes transparentes, spray formador de barreira e silicone sobre as proeminências ósseas, sob dispositivos médicos e em face. A posição recomendada é a do nadador, reposicionando o paciente a cada duas horas. Conclusão: a narrativa gráfica foi validada para enfermeiros experts cujo destaque está para os cuidados preventivos no sentido de promover o engajamento da equipe de Enfermagem frente à importância da prevenção da Lesão por Pressão no cuidado ao paciente com Coronavírus e pronado.
\end{abstract}

DESCRIPTORS: Lesão por pressão. COVID-19. Decúbito ventral. Cuidados de enfermagem. Prática avançada de enfermagem. Estomaterapia.

\section{PREVENTION OF PRESSURE INJURIES IN THE PRONATION OF COVID-19 PATIENTS: CONSTRUCTION OF A GRAPHIC NARRATIVE}

\begin{abstract}
Objective: build and validate the content of a graphic narrative on the prevention of Pressure Injury in the prone position in patients affected by the Coronavirus. Method: descriptive methodological study. For the construction of the graphic narrative, a scope review was carried out. Narrative content validation was performed by nurses working in stomatherapists in respiratory intensive care units in Santa Catarina, who responded to a form on Googleforms ${ }^{\circledR}$ with response options from disagree to agree. Data analysis was performed using the Content Validity Index, considering appropriate $>0.80 \%$. Results: the care listed to compose the narrative were: skin assessment; keeping the skin clean and hydrated; use of pressure redistribution devices; use of silicone foams,
\end{abstract}

1. Universidade Federal de Santa Catarina - Gestão do Cuidado em Enfermagem - Florianópolis (SC), Brasil.

2. Universidade Federal de Santa Catarina - Hospital Universitário - Secretaria Municipal de Saúde - Florianópolis (SC), Brasil.

3. Universidade Federal de Santa Catarina - Centro de Ciências da Saúde - Departamento de Enfermagem - Florianópolis (SC), Brasil.

4. Universidade Federal de Santa Catarina - Gestão do Cuidado em Enfermagem - Florianópolis (SC), Brasil.

*Autora correspondente: danielasoldera@hotmail.com

Editor de Seção: Manuela de Mendonça F Coelho

Recebido: Out. 04, 2021 | Aceito: Dez. 21, 2021

Como citar: Soldera D; Soares CF; Girondi JBR; Salum NC; Stein M; Amante LN; Sebold LF; Miranda GM. Prevenção de lesões por pressão na pronação de pacientes Covid-19: construção de uma narrativa gráfica. ESTIMA, Braz. J. Enterostomal Ther., 2021, 19: e2821. https://doi.org/10.30886/estima.v19.1136_PT 
transparent films, barrier-forming spray and silicone on bony prominences, under medical devices and on the face. The recommended position is that of the swimmer, repositioning the patient every two hours. Conclusion: the graphic narrative was valid for expert nurses whose emphasis is on preventive care in order to promote the engagement of the Nursing team in the face of the importance of Pressure Injury prevention in the care of patients with Coronavirus and prone.

DESCRIPTORS: Pressure injury. COVID-19. Prone. Nursing care. Advanced nursing practice. Stomatherapy.

\title{
PREVENCIÓN DE LESIONES POR PRESIÓN EN LA PRONACIÓN DE PACIENTES COVID-19: CONSTRUCCIÓN DE UNA NARRATIVA GRÁFICA
}

\begin{abstract}
RESUMEN
Objetivo: construir y validar el contenido de una narrativa gráfica sobre la prevención de Lesión por Presión en la posición prona en pacientes afectados por el Coronavirus. Método: estudio metodológico descriptivo. Para la construcción de la narrativa gráfica, se realizó la revisión del alcance. La validación de contenido de la narrativa fue realizada por enfermeros que trabajan en unidades de terapia intensiva respiratoria estomaterapeutas de Santa Catarina, quienes respondieron a un formulario en Googleforms ${ }^{\circledR}$ con opciones de respuesta de desacuerdo a acuerdo. El análisis de los datos se realizó por medio del Índice de Validez de Contenido, considerando adecuado $>0,80 \%$. Resultados: las precauciones enumeradas para componer esta narrativa fueron: evaluación de la piel; mantenimiento de la piel limpia e hidratada; utilización de dispositivos de redistribución de presión; utilización de espumas de silicona, películas transparentes, espray formador de barrera y silicona sobre las prominencias óseas, bajo dispositivos médicos y en la cara. La posición recomendada es la del nadador, reposicionando al paciente cada dos horas. Conclusión: la narrativa gráfica fue válida para enfermeros expertos cuyo énfasis está para los cuidados preventivos en el sentido de promover el compromiso del equipe de Enfermería frente a la importancia de la prevención de la Lesión por Presión en el cuidado al paciente con Coronavirus y propensos.
\end{abstract}

DESCRIPTORES: Lesión por presión. COVID-19. Decúbito ventral. Cuidados de enfermería. Práctica avanzada de enfermería. Estomaterapia.

\section{INTRODUÇÃO}

Em meados de março de 2020, a Organização Mundial de Saúde definiu situação pandêmica pelo aumento significativo de casos de COVID-19, uma doença infecciosa causada pelo novo Coronavírus (SARS-CoV-2)ㄹ.

Os pacientes acometidos pela COVID-19 podem ser assintomáticos, apresentar sintomas clínicos leves ou evoluir com quadro grave ou fatal caracterizado pela pneumonia com comprometimento respiratório severo, necessitando de internação em Unidade de Terapia Intensiva (UTI), evoluindo com intubação endotraqueal, instabilidade hemodinâmica e choque séptico².

Dentre as condutas adotadas para o tratamento da Síndrome do Desconforto Respiratório Agudo (SDRA), considera-se a manutenção do paciente na posição prona aplicada nas primeiras 48 horas, mantendo-o, no mínimo, 16 horas pronado. Tal condição aumenta significativamente o risco de desenvolver as Lesões por Pressão (LP) e as Lesões por Pressão Relacionadas a Dispositivos Médicos (LPRDM) ${ }^{3,4}$.

A National Pressure Injury Advisory Panel (NPIAP) alerta que o paciente com COVID-19 apresenta, pela fisiopatologia da doença, condições como coagulopatia sistêmica, hipercatabolismo e déficit nutricional, que o tornam mais suscetível ao aparecimento de lesões cutâneas, a saber, as LP5.

A motivação pela construção de uma narrativa gráfica embasada em evidências científicas sobre os cuidados preventivos de LP durante a posição prona do paciente com COVID-19 deu-se pela vivência na prática clínica e pelo desafio cotidiano em prover a melhor assistência para esse cuidado. Observa-se que as equipes de Enfermagem, neste momento, encontram-se exauridas e, muitas vezes, os cuidados com a pele e a prevenção de LP podem não estar sendo desenvolvidos na sua totalidade, 
seja por falta de tempo para a atualização desses conhecimentos ou pela sobrecarga de trabalho, afetando, inclusive, a labilidade emocional desses profissionais.

A agudização do cenário de dificuldades do processo de trabalho da Enfermagem na pandemia eleva o potencial de impacto na saúde mental dos trabalhadores decorrente do sofrimento, da escassez de Equipamentos de Proteção Individual (EPI), da fragilidade na descrição dos protocolos e fluxos para o controle efetivo de infecções, do prolongamento da jornada de trabalho, da formação profissional inadequada para o cenário de crise e de incertezas em relação às medidas terapêuticas ${ }^{6}$.

Nesse contexto, identificou-se, por meio do uso de tecnologias educativas, a oportunidade de contribuir para o aprendizado dos enfermeiros em linha de frente. $\mathrm{O}$ uso de recursos tecnológicos para fins pedagógicos configura-se em uma prática inovadora, que facilita e potencializa o processo de ensino e aprendizagem ${ }^{7}$.

Dentre as tecnologias existentes, as narrativas gráficas são conteúdos apresentados usando uma combinação de palavras e arte sequencial. As ilustrações de texto e imagem, cuidadosamente construídas, atuam como facilitadores no processo de aprendizagem ${ }^{8}$.

Nos últimos anos, a eficácia da educação baseada nas artes, em particular nos quadrinhos e novelas gráficas, tem sido cada vez mais estudada e identificada, em que a fusão de texto e imagem se mostra útil em um mundo orientado para a mídia9.

Vale ressaltar a importância do desenvolvimento de tecnologias educacionais elaboradas a partir de evidências científicas voltadas para a Enfermagem, sobretudo validadas por juízes. Nesse sentido, a validação de tecnologias educacionais fomenta a potencialidade de articulação da educação em saúde com as diversas tecnologias desenvolvidas, uma vez que o processo de validação averigua a capacidade de determinada tecnologia alcançar o objetivo a que se propõe ${ }^{10}$.

Frente a essas constatações, emergiu a pergunta de pesquisa: "Quais cuidados relacionados à prevenção de LP durante a pronação de pacientes com COVID-19 devem compor uma narrativa gráfica?”. A necessidade de se ter, acessíveis e de forma simplificada, as melhores evidências para o cuidado impulsionou as autoras para a elaboração desta tecnologia educativa a fim de que esse conhecimento seja amplamente divulgado, possibilitando que práticas seguras e eficazes sejam implementadas.

Este estudo teve como objetivo construir e validar uma narrativa gráfica sobre os cuidados preventivos de LP durante a pronação em pacientes acometidos pela COVID-19.

\section{MÉTODO}

Trata-se de um estudo metodológico. Esse teve como objetivo a construção e validação de uma tecnologia educativa, do tipo história em quadrinhos, com o intuito de contribuir para o aprimoramento dos conhecimentos da equipe de Enfermagem sobre os cuidados preventivos de LP durante a posição de prona em pacientes com COVID-19.

O produto foi desenvolvido no período de março a julho de 2021. As etapas para a construção foram: revisão de escopo, validação de conteúdo para compor a narrativa gráfica e validação da narrativa gráfica por enfermeiros de cuidados intensivos e/ou experts em Estomaterapia.

$\mathrm{Na}$ primeira etapa foi realizada a revisão de escopo para averiguar os cuidados preventivos de LP em pacientes com COVID-19 durante a posição prona. Para a construção da pergunta de pesquisa, utilizou-se a estratégia Population, Concept, Context (PCC), conforme recomendado pelo Joanna Briggs Institute (JBI) ${ }^{11}$, definindo-se: $\mathrm{P}$ - paciente com COVID-19; C - prevenção; C - assistência hospitalar. De agora em diante, a pergunta de pesquisa foi: "Quais as evidências científicas sobre a prevenção de LP em pacientes com COVID-19 pronados existem na literatura nacional e internacional?".

A busca deu-se nas bases de dados National Library of Medicine (PubMed), Scopus Review (Elsevier) e Scientific Electronic Library Online (SciELO), com recorte temporal do ano de 2020 a março de 2021, nos idiomas inglês, português e espanhol. Os seguintes descritores foram utilizados: "prone position"; "prone"; "proning”;"Hospital Care"; "Coronavirus Infection"; "Coronavirus Infections"; "pressure ulcer”; "Decubitus ulcer”. A revisão norteou a construção de conteúdo para compor a narrativa gráfica. Enquanto critérios de inclusão, definiram-se: estudos de pesquisas qualitativa 
e/ou quantitativas sobre o tema; ensaios; consensos publicados em periódicos das bases de dados selecionadas para o estudo; e estudos que continham os descritores e as palavras-chaves previstas no protocolo de busca. Foram excluídos estudos duplicados, cartas, editoriais, resumos publicados em anais de eventos e/ou periódicos, livros, dissertações, teses, programas, políticas de saúde, revisão de literatura, relatos de experiência, reflexão teórica ou que não atenda aos idiomas definidos.

A segunda etapa compreendeu a validação do conteúdo para compor a narrativa gráfica. Foi estruturado um formulário

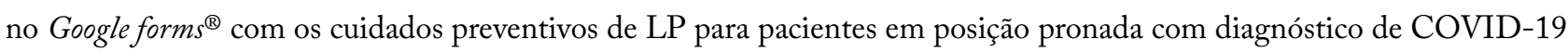
e requerida a validação quanto à concordância dos cuidados. Os dados obtidos na validação dos juízes foram agrupados e analisados utilizando o Índice de Validade de Conteúdo (IVC).

O IVC mede a proporção ou porcentagem de juízes em concordância sobre determinados aspectos de um instrumento e de seus itens ${ }^{11}$. Esse método consiste em uma escala de Likert com pontuação de 1 a 4 , em que: 1 = discordo; 2 = discordo parcialmente; 3 = concordo parcialmente; e 4 = concordo. Para calcular o IVC de cada item do instrumento, basta somar as respostas 3 e 4 dos participantes do comitê de especialistas e dividir o resultado dessa soma pelo número total de respostas ${ }^{12}$.

O tratamento estatístico considerou as seguintes categorias: completamente adequada e adequada para aqueles que obtiveram consenso $\geq 80 \%(0,8)$, sendo esse índice de concordância pautado em estudos de validação, uma vez que, para a verificação da validade do conteúdo na sua integralidade, a concordância mínima entre os juízes deve ser de 0,80 e, preferencialmente, superior a $0,90^{12}$.

Nessa etapa, foram convidados a participar, intencionalmente, 26 enfermeiros atuantes em instituições hospitalares da Grande Florianópolis, identificados por meio da técnica Bola de Neve, na qual um profissional com expertise em determinado tema indica outro e assim sucessivamente. Entretanto, participaram desta etapa 14 enfermeiros. O critério utilizado para a classificação dos experts foi a titulação em Terapia Intensiva e/ou Estomaterapia. Elencaram-se como critérios de exclusão: enfermeiros afastados no período de coleta de dados ou a não devolutiva em oito dias.

Após a validação de conteúdo, deu-se início à construção da história em quadrinhos por meio da ferramenta on-line Pixton $^{\circledR}$, que permite a criação desse tipo de tecnologia educativa.

A terceira etapa consistiu na validação de conteúdo da tecnologia educativa mediante a prévia apresentação da narrativa gráfica. Esta se deu por meio da estruturação de um novo formulário no Google forms ${ }^{\circledR}$, encaminhado aos examinadores que compuseram a etapa de validação de conteúdo anterior, seguindo os mesmos critérios de inclusão e exclusão. Propuseram-se a validação quanto à concordância, clareza, coerência, pertinência, objetividade ${ }^{13}$, bem como a avaliação acerca da apresentação do layout, a contribuição da tecnologia educativa para a prática assistencial do enfermeiro diante da prevenção de LP em pacientes com COVID-19 durante a posição prona e, por fim, opiniões e sugestões para aprimorar a narrativa gráfica.

O estudo foi aprovado pelo Comitê de Ética em Pesquisa com Seres Humanos (CEPSH) por meio do parecer consubstanciado CAAE no 12212519.2.0000.0121.

\section{RESULTADOS}

$\mathrm{Na}$ primeira etapa, foi realizada a revisão de escopo, que totalizou 107 artigos encontrados. Após a leitura dos títulos e resumos, foram selecionados 43 artigos para compor a construção da narrativa gráfica. Entre os estudos elencados, 12 foram excluídos por duplicidade e 26 por não contemplarem, na totalidade, o tema, estarem incompletos ou indisponíveis para acesso livre. Assim, a amostra final foi composta de cinco estudos elegíveis. Desses, três estudos apresentaram uma abordagem qualitativa ou quantitativa, um estudo era de coorte prospectivo e havia uma revisão de escopo. Três estudos foram publicados no ano de 2020 e dois, em 2021. Quanto ao idioma, a sua totalidade foi em inglês.

Os cuidados elencados na revisão de escopo compreenderam: avaliação da pele; utilização de dispositivos para a redistribuição de pressão sob proeminências ósseas; aplicação de spray formador de barreira e creme oftalmológico para a 
proteção ocular; manter a posição de nadador; reposicionar o paciente a cada duas horas; manter a pele limpa e hidratada; utilizar suportes para a redistribuição para aliviar pontos de pressão na face e no corpo; verificar a redistribuição de pressão em pontos exclusivos da posição prona e posicionamento de dispositivos médicos; aplicar curativos profiláticos de silicone sobre as proeminências ósseas e sob dispositivos médicos; avaliar a pele antes da pronação e após o reposicionamento para a posição supina; e aplicar curativos de hidrocoloides, espumas de cinco camadas, filmes transparentes e silicone para reduzir lesões na pele da face $4,15-18,20,22,23$.

$\mathrm{Na}$ segunda etapa foi validado o conteúdo da narrativa gráfica. A amostra foi por intencionalidade, composta por 14 enfermeiros avaliadores, predominantemente do sexo feminino ( $\mathrm{n}=13)$, todos titulados, em sua maioria, como mestres $(\mathrm{n}=8)$, seguidos por especialistas $(n=5)$ e um doutor. Em relação ao tempo de atuação na profissão, a maioria atuava entre 11 e 15 anos $(n=4)$. Quanto à área de atuação, a maior concentração foi no âmbito hospitalar ( $n=11)$ e em atividades relacionadas especificamente à Estomaterapia $(\mathrm{n}=3)$.

$\mathrm{Na}$ Tabela 1 são apresentadas as principais recomendações encontradas nos artigos e validadas pelos experts sobre os cuidados necessários antes de posicionar o paciente com COVID-19 em pronação.

Tabela 1. Principais recomendações antes de posicionar o paciente em prona. Florianópolis (SC), Brasil - 2021.

\begin{tabular}{|c|c|}
\hline CUIDADOS & IVC \\
\hline Avaliar a pele & 92,9 \\
\hline Utilizar dispositivos de redistribuição de pressão em proeminências ósseas & 92,9 \\
\hline Utilizar colchões apropriados & 100 \\
\hline Aplicar spray formador de barreira & 92,9 \\
\hline Aplicar creme oftalmológico para a proteção ocular e manter as pálpebras fechadas & 92,9 \\
\hline
\end{tabular}

IVC = Índice de Validade de Conteúdo.

$\mathrm{Na}$ Tabela 2 são apresentadas as principais recomendações encontradas nos artigos e validadas pelos experts sobre os cuidados necessários a serem realizados quando o paciente com COVID-19 estiver em pronação.

Tabela 2. Estratégias de implementação para a prevenção de Lesão por Pressão durante a prona. Florianópolis (SC), Brasil - 2021.

\begin{tabular}{|c|c|}
\hline CUIDADOS & IVC \\
\hline Manter a posição de nadador & 85,7 \\
\hline Reposicionar o paciente a cada duas horas & 92,9 \\
\hline Manter a pele limpa e hidratada & 100 \\
\hline Utilizar suportes para a redistribuição para aliviar pontos de pressão na face e no corpo & 100 \\
\hline $\begin{array}{l}\text { Verificar a redistribuição de pressão em pontos exclusivos da posição prona e posicionamento de } \\
\text { dispositivos médicos }\end{array}$ & 92,9 \\
\hline Utilizar curativos profiláticos de silicone sobre as proeminências ósseas e sob dispositivos médicos & 92,9 \\
\hline Avaliar a pele antes da pronação e após o reposicionamento para a posição supina & 100 \\
\hline $\begin{array}{l}\text { Aplicar curativos de hidrocoloides, espumas de cinco camadas, filmes transparentes e silicone para reduzir } \\
\text { lesões na pele da face }\end{array}$ & 92,9 \\
\hline
\end{tabular}

IVC = Índice de Validade de Conteúdo.

Após a validação dos cuidados relacionados à prevenção de LP durante a posição de prona do paciente com COVID19, foi construída a narrativa gráfica (Fig. 1, 2 e 3) utilizando a ferramenta Pixton ${ }^{\circledR}$. A narrativa está disponível na íntegra por meio do link: https://share.pixton.com/qr48ahb. 


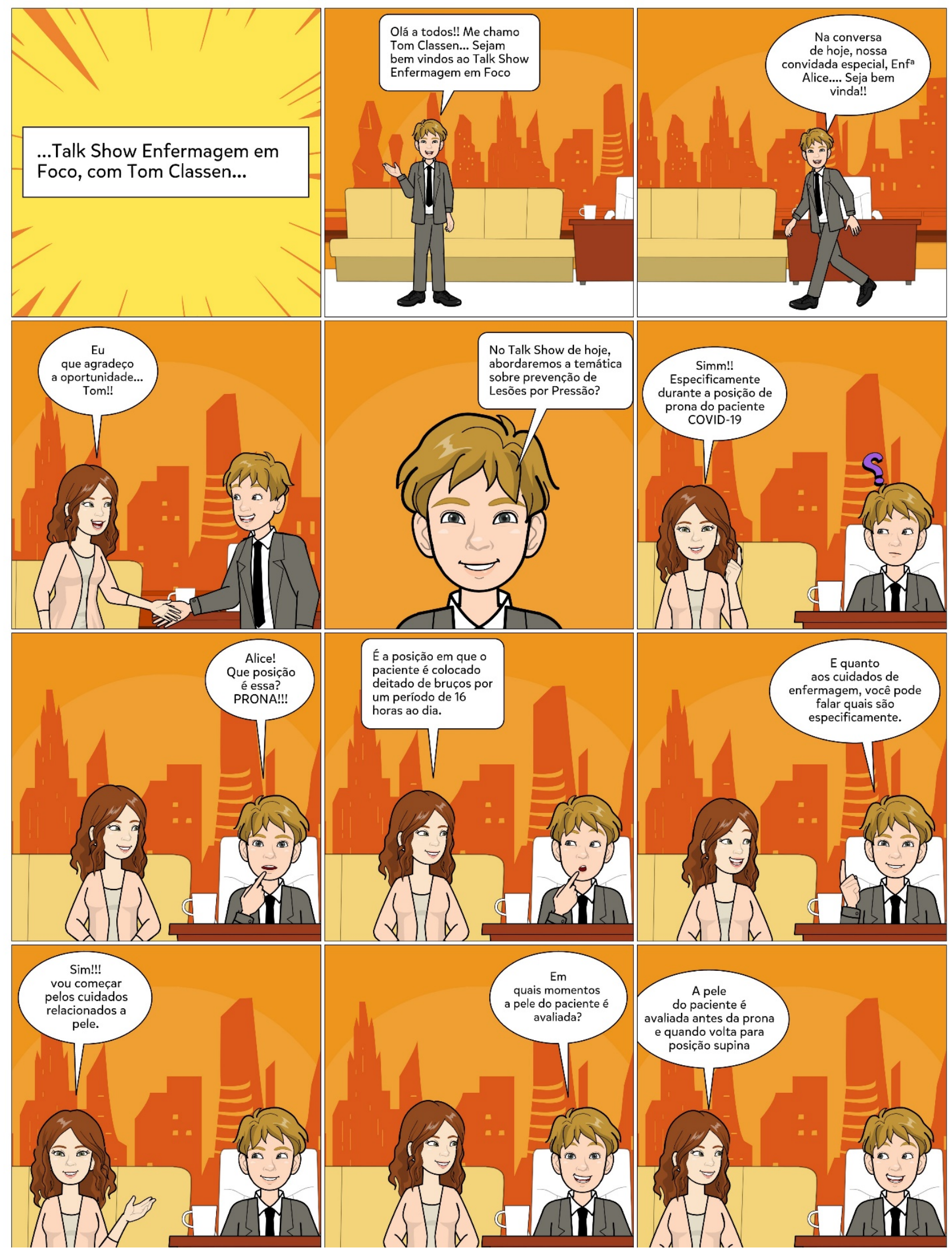

Figura 1. Telas da narrativa gráfica. Florianópolis (SC), Brasil - 2021. 


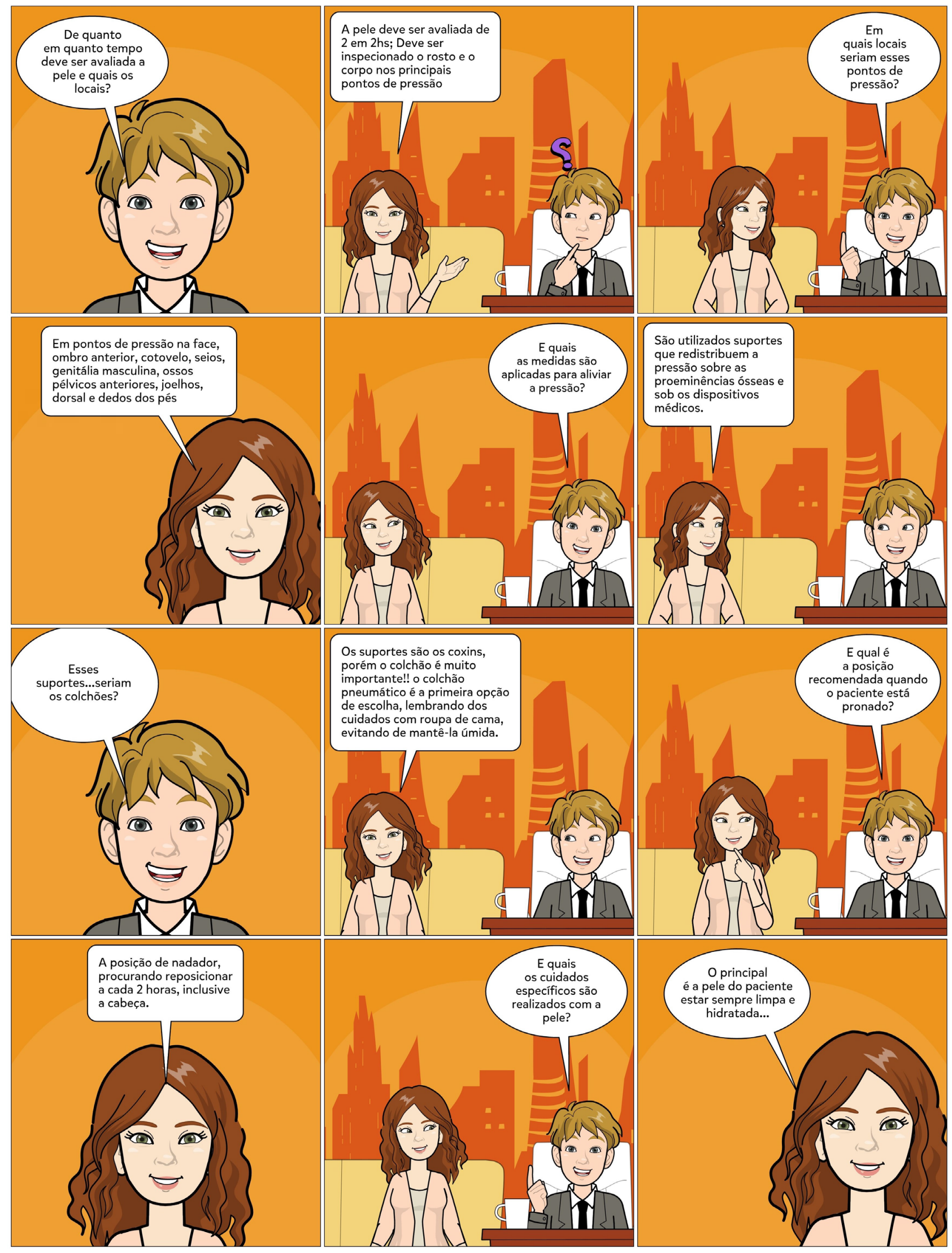

Figura 2. Telas da narrativa gráfica. Florianópolis (SC), Brasil - 2021. 

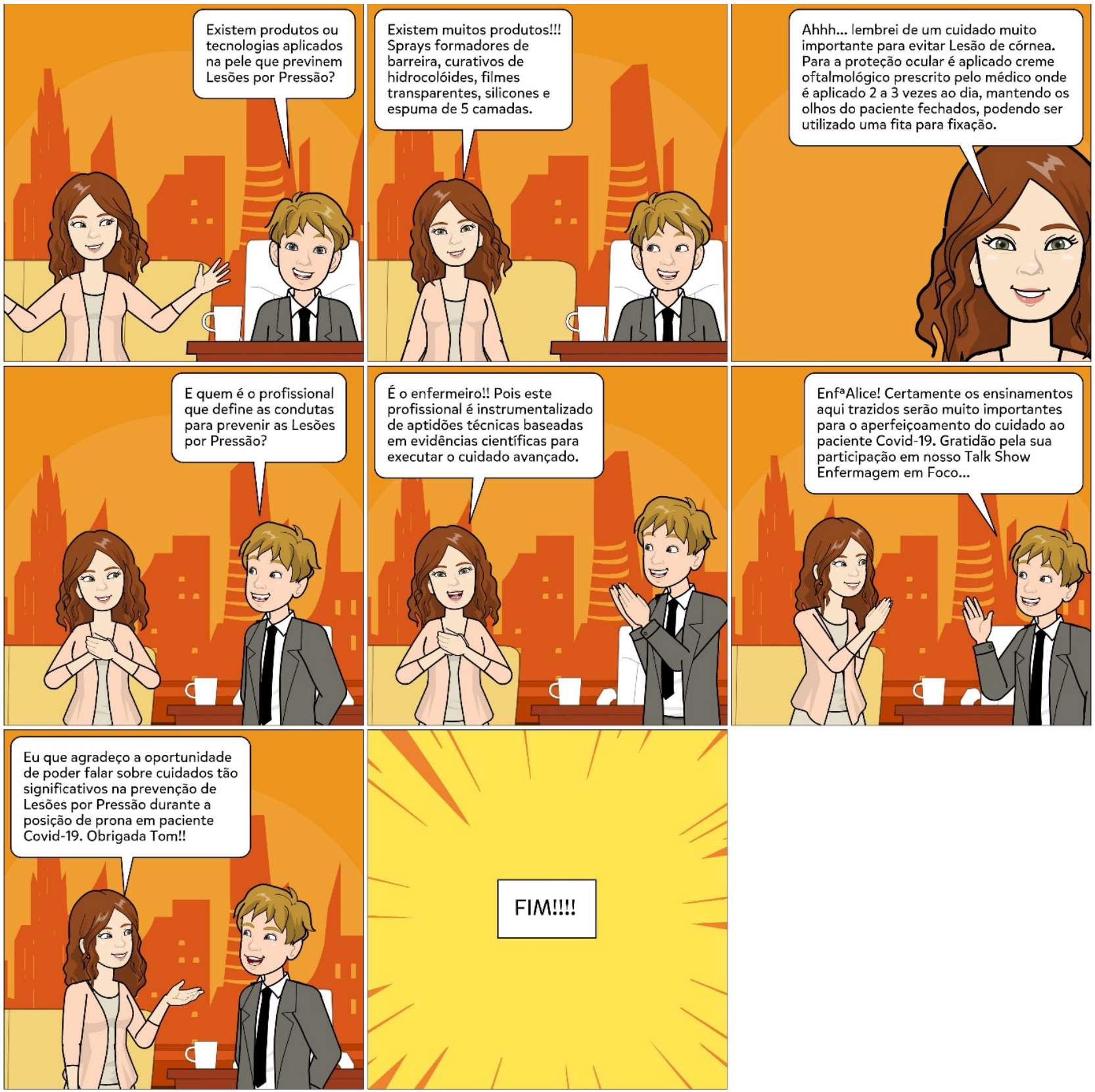

Figura 3. Telas da narrativa gráfica. Florianópolis (SC), Brasil - 2021.

$\mathrm{Na}$ terceira e última etapa foi realizada a validação da narrativa gráfica quanto à aparência e ao conteúdo pelos 14 enfermeiros avaliadores que participaram da segunda etapa, conforme os critérios de inclusão. O conteúdo foi apresentado aos experts por meio de um arquivo no qual pudessem avaliar, individualmente, cada uma das telas da narrativa gráfica quanto ao conteúdo dos diálogos, às imagens e características dos personagens e ao layout apresentado. Nessa etapa houve a concordância de $100 \%$ dos experts, tendo em vista que haviam realizado a validação acerca dos cuidados abordados na narrativa.

Quando questionados se a narrativa gráfica é considerada uma forma de disseminação de conhecimento de grande alcance, 12 consideram que sim e 14 consideram que a tecnologia educacional contribui para a prática assistencial do enfermeiro diante da prevenção de LP durante a posição prona.

Por fim, foi solicitado, aos examinadores, que avaliassem a tecnologia educativa elaborada, tecendo sugestões ou opiniões que pudessem contribuir para o aperfeiçoamento do material. Os achados estão descritos a seguir. 
Foi muito esclarecedor e detalbista [...] foi uma experiência rica e que conseguiu esclarecer algumas dúvidas que eu ainda tinha, como tempo para avaliação das lesões. Não fiquei com dúvidas. Excelente. (E1)

Muito didático!!! Utilizou as tecnologias para ensinar de modo divertido. (E3)

Atualmente, uma grande porcentagem da população tem acesso à internet através de um dispositivo móvel. A exemplo, os celulares promovem agilidade e facilidade na comunicação e, para quem quer fazer bom uso desta tecnologia, adquirir conhecimento através de uma narrativa gráfica é inovador. A transposição do texto verbal para o texto visualé um método eficiente de comunicação e aprendizado. (E4)

Conteúdo bem didático. Seria interessante ressaltar que, em pacientes obesos e edemaciados, os cuidados devem ser mais intensivos, pois, nestas condiçôes, o aparecimento da LP é maior, principalmente por dispositivos médicos. (E5)

Poderia ser uma linguagem mais coloquial para, realmente, disseminar conhecimento. (E7)

Excelente produto. Forma atrativa e objetiva de capacitação. (E8)

Ao término da validação da narrativa gráfica pelos experts, foi solicitado o registro de direito autoral na Câmara Brasileira do Livro.

\section{DISCUSSÃO}

Este estudo foi estruturado a partir da revisão de escopo acerca dos cuidados de Enfermagem para a prevenção de LP durante a posição prona em pacientes acometidos pela COVID-19 e validados por enfermeiros atuantes em UTI COVID e/ou estomaterapeutas experts em cuidados com a pele para, então, prosseguir com a construção da narrativa gráfica.

A pronação do paciente com COVID-19 auxilia o tratamento da SDRA, portanto, é necessário que permaneça até 16 horas por dia nessa posição, alternando-a a cada 8 horas para decúbito dorsal, embora essa posição aumente o risco de $\mathrm{LP}^{14}$.

Pacientes com instabilidade hemodinâmica e/ou respiratórias atendidos em UTIs apresentam um quadro clínico de risco para o desenvolvimento de LP decorrente do uso de drogas vasoativas, sedação, ventilação mecânica invasiva, entre outros $^{15}$. Assim, podem apresentar deterioração da integridade cutânea em questão de horas. Além dos pontos de pressão natural no corpo decorrentes do decúbito proposto, outro fator agravante são os múltiplos dispositivos médicos utilizados ${ }^{16}$.

Nesse contexto, a prevenção de LP configura-se como um desafio para a Enfermagem, pois as alterações decorrentes da infecção expõem o paciente à maior instabilidade, menor oxigenação tecidual, tempo de internação em unidade crítica prolongada e dificuldade de reposicionamento ${ }^{16}$.

O enfermeiro, com a autonomia e a capacidade de tomada de decisão para o cuidado avançado voltado à prevenção desse agravo, deve realizar, primeiramente, a avaliação da pele do paciente antes da posição prona, para que possa aplicar as condutas necessárias como a utilização de dispositivos ou suportes que redistribuam e aliviem a pressão em proeminências ósseas, bem como a escolha de colchões apropriados para reduzir a incidência desse agravo ${ }^{4}$.

Ao enfatizar o cuidado com os olhos, a pressão direta nas órbitas, juntamente às alterações vasculares, provoca impacto muscular extraocular, desencadeando edema conjuntival, hemorragia e lesão na córnea, o que já é possível de ser observado, aproximadamente, dez minutos após esse posicionamento ${ }^{17}$.

A posição de escolha durante a posição prona é a de nadador para que o rosto fique voltado em direção ao braço flexionado e o outro braço permaneça voltado para trás. Essa posição permite a movimentação da cabeça, do tubo endotraqueal e da sonda nasoenteral ao mesmo tempo e deve ser realizada a cada duas horas ${ }^{4,18}$. Ainda, recomenda-se a realização de pequenos reposicionamentos a cada duas ou quatro horas, utilizando-se, para tal, a técnica de reposicionamento nadador, na qual se alterna a posição dos braços e da cabeça ${ }^{16}$. 
No que tange ao reposicionamento, a mobilização precoce torna-se uma prioridade, pois se deve considerar a fisiopatologia da SARS-CoV-2 em relação ao risco de trombose grave. A posição e a duração desse posicionamento, portanto, necessitam ser documentadas ${ }^{19}$. Além de reduzir complicações vasculares, o reposicionamento e o alívio da pressão são intervenções de Enfermagem que reduzem o risco de LP. Portanto, cabe ao enfermeiro com sua expertise coordenar a equipe durante o reposicionamento, uma vez que, para prestar esse cuidado, muitas vezes, são envolvidos, em média, de seis a sete profissionais ${ }^{20,21}$.

Nesse sentido, a primeira preocupação é com a pele, que deve ser mantida limpa e hidratada, pois a umidade provoca maceração dos tecidos, potencializando o risco de LP. Sobre a limpeza, o recomendado é usar sabonetes para o banho com $\mathrm{pH}$ levemente acidificado, o qual se aproxima do $\mathrm{pH}$ fisiológico da pele. Outros tipos de sabonete e antissépticos não são recomendados pelo risco de causarem irritação e ressecamento da pele $e^{4,16,22}$.

Em relação à umidade, a atenção deve ser redobrada com pacientes portadores de incontinência (urina e/ou fezes), de lesões exsudativas, de estomas com vazamentos, dentre outras situações. Nesses casos, além da constante inspeção, recomenda-se a utilização de produtos de proteção de barreira, associados a produtos prontos sem enxague para a limpeza da pele da área perigenital e adjacências, com surfactantes e $\mathrm{pH}$ ácido ${ }^{23}$.

Destaca-se a importância da avaliação e adequada adaptação da superfície de apoio, bem como de coxins e travesseiros ${ }^{24}$. Os principais pontos de pressão nesse posicionamento são: testa, bochecha, queixo, clavícula, cotovelo, região inframamária, genitais, pelve, joelhos, dorso e dedos dos pés e regiões adjacentes aos dispositivos médicos ${ }^{16}$.

Outras medidas essenciais devem ser adotadas, abrangendo a avaliação clínica global do paciente e intervenções adequadas, para o manejo das intercorrências. Ainda são importantes cuidados quanto ao rodízio de sítio do oxímetro e dos demais dispositivos sempre que possível ${ }^{16}$.

A avaliação da pele antes e após a posição prona deve ser um cuidado minucioso realizado pelo enfermeiro para avaliar a qualidade do cuidado prestado e quanto às medidas preventivas, se foram efetivas ou não. A inspeção da pele diária é necessária, tendo em vista a rápida mudança de fatores de risco nesses pacientes agudos, como o caso dos infectados pela COVID-19. Para a redistribuição de pressão, além dos já mencionados anteriormente, pode ser utilizada espuma de silicone em pontos em que há pressão sobre proeminências ósseas ${ }^{22}$. Ainda, para auxiliar no alívio da pressão e redução da fricção da pele com a interface dos dispositivos médicos, pode-se lançar mão de espuma de silicone para reduzir o risco de $\mathrm{LP}^{16,25}$.

Nesse processo, cabe aos enfermeiros executar os cuidados avançados em Enfermagem, de modo a prevenir o desenvolvimento de LP durante a posição prona em pacientes com COVID-19, principalmente em pacientes obesos e edemaciados, pois após o desenvolvimento de LP, esta torna-se onerosa para os sistemas de saúde, podendo interferir na recuperação do paciente, aumentando o tempo de internação hospitalar e com risco potencial de complicações fatais por sepse 2 .

O NPIAP ${ }^{4}$ reforça a importância não só de garantir a implementação das práticas aqui relatadas, mas, também, que todas as intervenções de Enfermagem sejam devidamente executadas e registradas nos prontuários dos pacientes, alertando que, desse modo, se pode concluir que, caso o paciente desenvolva LP, esta poderá ser considerada inevitável ${ }^{16}$.

Por fim, destaca-se que narrativas gráficas ainda são pouco exploradas como recurso acadêmico, especialmente na Enfermagem. Por esse motivo, o artigo objetivou apresentar essa ferramenta enquanto possibilidade de gerar aprendizado rápido, prático e objetivo, trazendo um maior envolvimento do leitor com o instrumento e, sobretudo, a facilidade de divulgação por meio das mídias digitais.

\section{Limitações do estudo}

Como limitação, considera-se a validação com experts de uma determinada região geográfica, o que pode retratar parte da problemática estudada.

\section{Contribuições para a prática}

Enquanto contribuições para a prática, o modo como foi estruturado o conteúdo científico por meio de uma narrativa gráfica é uma inovação tecnológica para a área da Enfermagem, em vista da possibilidade de divulgação em grande escala por meio das mídias sociais. 


\section{CONCLUSÃO}

Ao considerar o contexto da Prática Avançada em Enfermagem, na qual ocorre a integração entre os pilares de educação, pesquisa, prática assistencial e gestão para o desenvolvimento profissional, a temática elucidada contempla um cuidado avançado de Enfermagem, pois o enfermeiro desenvolve competências e tem autonomia e tomada de decisões para desempenhar os cuidados preventivos de LP durante a pronação dos pacientes, principalmente em ambiente de cuidados intensivos.

O objetivo proposto de validação dos cuidados preventivos com enfermeiros intensivistas e/ou estomaterapeutas foi alcançado, havendo um consenso sobre as condutas desenvolvidas por ambas as especialidades. O desenvolvimento da narrativa gráfica sobre cuidados preventivos busca a promoção e o engajamento da equipe de Enfermagem frente à importância da prevenção da LP enquanto o paciente permanece em posição de pronação.

Considera-se a narrativa gráfica uma tecnologia inovadora dentre os produtos desenvolvidos na área da Enfermagem, a qual tem como intuito demonstrar, para a comunidade acadêmica e assistencial, que se pode sim utilizar a ludicidade para aprender e ensinar.

\section{CONTRIBUIÇÃO DOS AUTORES}

Conceitualização: Soldera D e Girondi JBR; Metodologia: Soldera D e Girondi JBR; Investigação: Soldera D e Girondi JBR; Redação - Primeira versão: Soldera D, Girondi JBR, Soares CF, Amante LN, Sebold LF, Salum NC, Stein M e Miranda GM; Redação - Revisão \& Edição: Soldera D, Girondi JBR, Soares CF, Amante LN, Sebold LF, Salum NC, Stein M e Miranda GM.

\section{DISPONIBILIDADE DE DADOS DE PESQUISA}

Todos os dados estão presentes no artigo.

\section{FINANCIAMENTO}

Não se aplica.

\section{AGRADECIMENTOS}

Não se aplica.

\section{REFERÊNCIAS}

1. World Health Organization. Rational use of personal protective equipment for coronavirus disease (COVID-19): Interim guidance. World Health Organization. 2020. [citado 2021 Mar 21]. Disponível em: https://apps.who.int/iris/bitstream/ handle/10665/331215/WHO-2019-nCov-IPCPPE_use-2020.1-eng.pdf

2. Associação de Medicina Intensiva Brasileira. Coronavírus: esclarecimentos da AMIB pelo Comitê de Sepse e Infecção. 2020. São Paulo: AMB (SP); 2020. Disponível em: https://www.amib.org.br/fileadmin/user_upload/amib/2020/marco/21/11_ CORONAVIRUS_Esclarecimentos_da_AMIB_pelo_Comite_de_Sepse_e_Infeccao.pdf

3. Véras JB, Martinez BP, Neto MG, Saquetto MB, Conceição CS, Silva CM. Efeitos da posição prona em pacientes com síndrome do desconforto respiratório agudo: uma revisão sistemática. Rev Pesqui Fisioter 2019;9(1):129-138. https://doi.org/10.17267/22382704rpf.v9i1.2175

4. European Pressure Ulcer Advisory Panel, National Pressure Injury Advisory Panel and Pan Pacific Pressure Injury Alliance. Prevention and Treatment of Pressure Ulcers/Injuries: Quick Reference Guide. Emily Haesler (Ed.). EPUAP/NPIAP/PPPIA. 2019. [citado 2021 Mar 20]. Disponível em: http://www.internationalguideline.com/static/pdfs/Quick_Reference_Guide-10Mar2019. pdf 
5. Black J, Cuddigan J, Capasso V, Cox J, Delmore B, Munoz N et al. On behalf of the National Pressure Injury Advisory Panel. Unavoidable Pressure Injury during COVID-19 Crisis: A Position Paper from the National Pressure Injury Advisory Panel. 2020. [citado 2021 Mar 20]. Disponível em: https://cdn.ymaws.com/npiap.com/resource/resmgr/white_papers/Unavoidable_in_ COVID_Pandemi.pdf

6. Souza NVDO, Carvalho EC, Soares SSS, Varella TCMML, Pereira SRM, Andrade KBS. Nursing work in the COVID-19 pandemic and repercussions for workers' mental health. Rev Gaúcha Enferm. 2021;42(spe):e20200225. https://doi.org/10.1590/19831447.2021.20200225

7. Machado GB, Wives LK, Grandi R. As comunidades de prática como ferramenta para formação continuada de professores: percepções docentes sobre o uso da tecnologia para compartilhamento e aprimoramento das práticas pedagógicas. Anais do XXX SBIE. 2019; 1991-2004. https://doi.org/10.5753/cbie.sbie.2019.1995

8. Green MJ, Myers KR. Medicina gráfica: uso de quadrinhos na educação médica e no atendimento ao paciente. BMJ. 2010; 340:c863. https://doi.org/10.1136/bmj.c863

9. Albright KS, Gavigan, K. Information Vaccine: Using Graphic Novels as an HIV/AIDS Prevention Resource for Young Adults. J Educ Libr Inf Sci. 2014;55(2) [Citado 2021 Nov 15]: 178-85. Disponível em: https://files.eric.ed.gov/fulltext/EJ1074316.pdf

10. Cassiano AN, Silva CJA, Nogueira ILA, Elias TMN, Teixeira E, Menezes RMP. Validação de tecnologias educacionais: estudo bibliométrico em teses e dissertações de enfermagem. Revista de Enfermagem do Centro-Oeste Mineiro. 2020;10:e3900. http://doi.org/10.19175/recom.v10i0.3900

11. The Joanna Briggs Institute. Joanna Briggs Institute Reviewers manual: 2014 edition: methodology for jbi mixed methods systematic reviews. 2014 [citado 2021 Mar 20]. Disponível em: https://nursing.Isuhsc.edu/JBI/docs/ReviewersManuals/MixedMethods.pdf

12. Alexandre NMC, Coluci MZO. Validade de conteúdo nos processos de construção e adaptação de instrumentos de medidas. Ciênc Saúde Coletiva. 2011;16(7):3061-68. https://doi.org/10.1590/S1413-81232011000800006

13. Pasquali, L. Psicometria. Revi Esc Enferm. 2009; 43(Esp.):992-9. https://doi.org/10.1590/S0080-62342009000500002

14. Dondorp AM, Hayat M, Aryal D, Beane A, Schultz MJ. Respiratory Support in COVID-19 Patients, with a Focus on ResourceLimited Settings. Am J Trop Med Hyg. 2020;102(6):1191-7. https://doi.org/10.4269/ajtmh.20-0283

15. Souza MFC, Zanei SSV, Whitaker IY. Risco de lesão por pressão em UTI: adaptação transcultural e confiabilidade da EVARUCI. Acta Paul Enferm. 2018;31(2):201-8. https://doi.org/10.1590/1982-0194201800029

16. Ramalho AO, Freitas PSS, Moraes JT, Nogueira PC. Reflexões sobre as recomendações para prevenção de lesões por pressão durante a pandemia de COVID-19. ESTIMA, BrazJ Enterostomal Ther. 2020;18:e2520. https://doi.org/10.30886/estima.v18.940_ PT

17. Saran S, Gurjar M, Kanaujia V, Ghosh PS, Gupta A, Mishra P et al. Effect of Prone Positioning on Intraocular Pressure in Patients With Acute Respiratory Distress Syndrome. Crit Care Med. 2019;47(9):e761-6. https://doi.org/10.1097/CCM.0000000000003893

18. Wounds International TV. COVID-19 and tips for safe skin and proning: What should skin care involve for patients in the prone position?. 2020. [citado 2021 Mar 20]. Disponível em: https://woundsinternationaltv.com/editors-chioce/covid-19-and-tips-forsafe-skin-and-proning/

19. Magro C, Mulvey JJ, Berlin D, Nuovo G, Salvatore S, Harp J et al. Complement associated microvascular injury and thrombosis in the pathogenesis of severe COVID-19 infection: A report of five cases. Transl Res. 2020;220:1-13. https://doi.org/10.1016/j. trsl.2020.04.007

20. Odor PM, Neun M, Bampoe S, Clark S, Heaton D, Hoogenboom EM et al. Anaesthesia and COVID-19: infection control. Br J Anaesth. 2020;125(1):16-24. https://doi.org/10.1016/j.bja.2020.03.025

21. Lou B, Li TD, Zheng SF, Su YY, Li ZY, Liu W et al. Serology characteristics of SARS-CoV-2 infection after exposure and postsymptom onset. Eur Respir J. 2020;56(2): 2000763. https://doi.org/10.1183/13993003.00763-202

22. Black, J, Alves P, Brindle CT, Dealey C, Santamaria N, Call E et al. Use of wound dressings to enhance prevention of pressure ulcers caused by medical devices. Int Wound J. 2015;12(3):322-7. https://doi.org/10.1111/iwj.12111

23. Payne D. Skin integrity in older adults: pressure-prone, inaccessible areas of the body. Br J Community Nurs. 2020;25(1):22-6. https://doi.org/10.12968/bjcn.2020.25.1.22

24. Moore Z, Patton D, Avsar P, McEvoy NL, Curley G, Budri A et al. Prevention of pressure ulcers among individuals cared for in the prone position: lessons for the COVID-19 emergency. J Wound Care. 2020;29(6):312-20. https://doi.org/10.12968/ jowc.2020.29.6.312

25. Kim RS, Mullins K. Preventing Facial Pressure Ulcers in Acute Respiratory Distress Syndrome (ARDS). J Wound Ostomy Continence Nurs. 2016;43(4):427-9. https://doi.org/10.1097/WON.0000000000000247 